\section{A program to compute nonparametric partial correlation coefficients}

\author{
CARL CRAIG MORRIS \\ Baylor University, Waco, Texas
}

Measures of correlation and partial correlation provide crucial information about the interrelations among research variables and underlying factors. The techniques of causal modeling and causal path analysis, in which measures of partial association carry the critical information, provide a powerful quantitative framework within which to develop and test scientific theories (Asher, 1983; Cohen \& Cohen, 1983; Kim \& Mueller, 1978; Morris, 1990a, 1990b; Reynolds, 1974). The paper by Reynolds (1974) is provocative in that it demonstrates the efficacy of nonparametric (ordinal) partial correlation coefficients in testing spurious correlation models. To support population inferences, parametric measures of association or partial association, such as the (partial) Pearson product moment correlation or the partial regression coefficient, require stringent structural assumptions (general linear model), distributional assumptions (bivariate normal distribution), and, according to some (Nelson, 1984), scaling assumptions (interval or ratio scale). Measurement in the social sciences often fails to satisfy some or all of these assumptions, which renders parametric measures of association questionable (Nelson, 1984; Reynolds, 1974). To be sure, the debate about measurement scales and scale-dependent "permissible transformations" continues (Gaito, 1980; Townsend \& Ashby, 1984), and sophisticated qualifications of earlier conclusions have recently emerged (Davison \& Sharma, 1988; Michell, 1986). Nevertheless, nonparametric measures obviate parametric assumptions, yet nonparametric measures of correlation and partial correlation are almost completely absent from the experimental literature. The present scarcity of nonparametric measures of partial correlation in the literature may be due to their unfamiliarity and inaccessibility relative to the more popular parametric measures.

My goal in the present paper is to make nonparametric measures of correlation and partial correlation more accessible to researchers by describing the statistical basis of three related nonparametric measures and presenting a computer program that computes all three. First, following Wilson (1974), I describe a class of three nonparametric bivariate correlations. Next, I describe Quade's (1974) generalization of this class of correlations to partial correlations and his formula for calculating their asymptotic standard error and thereby testing their statistical significance. Finally, I present a flexible PC-compatible computer program (Turbo Pascal version 3.0) that computes

\footnotetext{
The author's mailing address is: Behavioral Statistics Program, Department of Psychology, Baylor University, Waco, TX 76798.
}

all three measures, their standard errors, and $Z$ statistics for asymptotic tests of statistical significance.

Correlational data necessarily consist of a set of $n$ cases of the form $\left(x_{i}, y_{i}\right)$, where $i=1,2, \ldots(j) \ldots(k) \ldots n$. Now if any two distinct cases $\left(x_{j}, y_{j}\right),\left(x_{k}, y_{k}\right)$ are considered, several possible relations between the pair may obtain. If $x_{j}<x_{k}$ and $y_{j}<y_{k}$, or if $x_{j}>x_{k}$ and $y_{j}>y_{k}$, then the pair is a concordant pair. If, however, $x_{j}<x_{k}$ and $y_{j}>y_{k}$, or if $x_{j}>x_{k}$ and $y_{j}<y_{k}$, then the pair is a discordant pair. Finally, the pair may be tied on only $X$, only $Y$, or both $X$ and $Y$, yielding three distinct types of tied pairs. Each of the three nonparametric correlations to be described assesses the relation between $X$ and $Y$ by computing a ratio of the form $(C-D) / R$, where $C$ and $D$ are the numbers of concordant and discordant pairs, and where $R$ is the total number of relevant pairs. To the extent that concordant pairs prevail, then $C-D$ will be positive, implying a positive relation between $X$ and $Y$; conversely, a prevalence of discordant pairs means that $C-D$ will be negative, implying a negative relation between $X$ and $Y$. If neither a positive nor a negative relation prevails, then concordant and discordant pairs will be equiprobable, and $C-D$ will approach zero. All three measures consider concordant and discordant pairs relevant and hence include $C+D$ in $R$, but they differ in whether ties are counted as relevant. In the following, the number of cases tied only on $X$ is denoted $T_{x}$, the number of cases tied only on $Y$ is denoted $T_{y}$, and the number of cases tied on hoth $X$ and $Y$ is denoted $T_{x y}$. The Goodman-Kruskal (1954) gamma statistic is $(C-D) /(C+D)$ and thus ignores ties. Somer's (1962) $d_{y x}$ statistic is $(C-D) /\left(C+D+T_{y}\right)$, which considers ties on $Y$, but not $X$, relevant; note that $d_{y x}$ is an asymmetrical measure; that is, it may differ, depending on which variable is being predicted by the other. Wilson's (1974) $e$ statistic is $(C-D) /\left(C+D+T_{x}+T_{y}\right)$, which considers ties on both $X$ and $Y$ relevant. All three coefficients range from -1 to 1 , with negative values indicating negative relationships and positive values indicating positive relationships. Wilson (1974) contends that each measure assesses a distinct hypothesis about the type of bivariate relationship, but that discussion is beyond the scope of this paper. Note that when there are no ties on either $X$ or $Y$, all three measures yield the same result.

Quade (1974) showed that for bivariate ordinal correlations of the form $(C-D) / R$, as defined above, there is a form of computation that permits both derivation of the standard error of the coefficient and generalization to a partial correlation coefficient. For each case $i$ in the total set of data, $R_{i}$ is the number of relevant pairs that include the observed case $\left(x_{i}, y_{i}\right)$, and $W_{i}$ is the number of concordant pairs minus the number of discordant pairs in that subset of relevant pairs. Across all cases, each pair is counted twice $(i, j$ and $j, i)$, so $\sum W_{i}=2 W=2\left(C_{i}-D_{i}\right)$ and $\sum R_{i}=2 R$. Hence, the ratio $\left(\sum W_{i}\right) /\left(\Sigma R_{i}\right)$ is a weighted average measure, with $R_{i}$ defined as appropriate for the 
desired statistic. The asymptotic standard error of this ratio is a function of the $W_{i}$ and $R_{i}$, and as the number of cases $n$ increases, the sampling distribution of the ratio of the statistic to its standard error approaches the standard normal distribution, thereby permitting a test of statistical significance for the coefficient. The equation Quade provides for computing the asymptotic standard error of this class of nonparametric correlation coefficients is

$S E=\frac{2}{\left[\sum R_{i}\right]^{2}} \sqrt{\left[\sum R_{i}\right]^{2} \sum W_{i}^{2}-2 \sum R_{i} \sum W_{i} \sum R_{i} W_{i}+\left[\sum W_{i}\right]^{2} \sum R_{i}^{2}}$.

Now consider that correlational data often involve related sets of observations in which more than two observations per case are considered. For example, the interrelations among three variables may be of interest, with each case providing a related triple of the form $\left(X_{i}, Y_{i}, Z_{i}\right)$. The question is how $X$ and $Y$ are related when $Z$ is controlled; that is, what is the partial correlation between $X$ and $Y$ with the effects of $Z$ statistically controlled? When only two variables are considered, Quade's formula, $\left(\sum W_{i}\right) /\left(\Sigma R_{i}\right)$, yields the same statistic as the original formulas described above while also permitting derivation of their standard errors. Quade's formula permits another crucial generalization, however. By including in $R_{i}$ only those pairs of cases for which some third variable $Z$ is relatively constant, one obtains a weighted average correlation of $X$ and $Y$ with $Z$ controlled. A "tolerance" value $\mathrm{L}$ must be defined for the variable to be controlled. That is, only those pairs $R_{i}$ for which $\left(Z_{i}-Z_{j}\right) \leq L_{z}$ are considered relevant for each case $i$, where the experimenter defines the acceptable tolerance limit. For example, setting $L_{z}$ to zero limits $R_{i}$ to only those pairs of cases with identical values on variable $Z$. This method of partialing extends easily to any number of control variables. For example, with four variables, $a, b, c, d$, the correlation between $a$ and $b$ is computed by determining $W_{i}$ and $R_{i}$ for each case $\left(a_{i}, b_{i}, c_{i}, d_{i}\right)$ such that for all pairs of cases, $\left(c_{i}-c_{j}\right) \leq L_{c}$ and $\left(d_{i}-d_{j}\right) \leq L_{d}$, and so forth. Regardless of the statistic being computed, $g, d_{y x}$, or $e$, the procedure is to compute the ratio $\left(\sum W_{i}\right) /\left(\sum R_{i}\right)$, its standard error, and their ratio, using any standard $z$ table to determine the statistical significance of the coefficient.

I now describe a Turbo-Pascal (version 3.0) PC program that computes all three of the nonparametric (partial) correlations described above, their standard errors, and $z$ statistics for tests of statistical significance using the standard normal $z$ distribution. The program is called OrdCorr, short for "ordinal correlation." The program should be compiled as a .com file. The program can be run either interactively, in which case no parameters must be specified upon execution, or by providing two parameters upon execution: an input filename followed by an output filename. The input and output filenames are specified by the user. The syntax for executing OrdCorr is OrdCorr [infile] [outfile], where [infile] is the text file containing program control information (and the data, unless the data are in a separate file), and [outfile] receives the results of the analysis. The routine
GetParameters evaluates the parameter list, if any. If there are no parameters, the routine MainMenu is executed to permit interactive use of the program. Once control and data files have been created either interactively or via a standard text editor, ReadData reads in the control and data file(s), RankTransform rank transforms the data if so desired, and ComputeCorr computes the statistical analyses and writes the output to the designated output file, which may also be to the screen or printer if so desired.

The operation of the program can be outlined by referring to the example input control file in Table 1. Note that there is an asterisk $\left(^{*}\right)$ near the top of the file in the leftmost column. The program ignores all information above the asterisk, so any number of lines of descriptive information can appear above it. The program control information begins on the line following the asterisk. Each line of control information contains an explanatory comment to the right of the control information; this commentary information can be changed or deleted without affecting the program. When this control file is created interactively, rather than directly via a text editor, the commentary information is omitted. Line 1 of control information is the name of the file containing the raw data to be analyzed. This file may be the control file or another file. If it is the control file name, then the data file should be appended to the end of the control file. When a data file is created interactively, it is not appended to the control file. Any standard text editor can be used to simply append the data file to the end of the control file if that is desired. In any case, an asterisk must also appear in the leftmost column on the line immediately above the first line of data. As with the asterisk preceding the first line of control information, any number of lines containing any sort of text can appear before the asterisk, allowing descriptive information (or the control file statements) to appear above the data. Columns of data in the data file correspond to variables, and rows correspond to cases. An example data file appears in Table 2 .

Line 2 of control information specifies the number of variables that appear in the data file-that is, the number

Table 1

Example Program Control File

\begin{tabular}{|c|c|}
\hline oc.cs & \\
\hline Nonparame & Partial Correlation Analysis \\
\hline$*$ & \\
\hline oc.data & Data filename \\
\hline 5 & Number of variables in data file \\
\hline 11111 & Rank transform per variable? $(1=$ yes, $0=$ no $)$ \\
\hline 33333 & Matching tolerance (per variable) \\
\hline 10 & Number of cases \\
\hline 0 & Data output ( $1=$ full, $0=$ summary) \\
\hline 6 & Number of correlations to compute \\
\hline 12 & Correlations to compute \\
\hline 12.3 & \\
\hline 12.34 & \\
\hline 12.345 & \\
\hline 31.245 & \\
\hline 54 & \\
\hline
\end{tabular}


Table 2

Example Data File

\begin{tabular}{|c|c|c|c|c|c|}
\hline $\begin{array}{l}\alpha, \\
*\end{array}$ & & & & & \\
\hline I & 1 & 9 & 3 & 4 & 5 \\
\hline 2 & 5 & 6 & 7 & 8 & 9 \\
\hline 3 & 1 & 5 & 9 & 2 & 9 \\
\hline 4 & 8 & 7 & 9 & 3 & 6 \\
\hline 5 & 0 & 5 & 4 & 6 & 3 \\
\hline 6 & 8 & 7 & 9 & 7 & 8 \\
\hline 7 & 0 & 9 & 2 & 1 & 2 \\
\hline 8 & 2 & 4 & 1 & 8 & 5 \\
\hline 9 & 1 & 5 & 9 & 3 & 9 \\
\hline 10 & 9 & 2 & 4 & 5 & 9 \\
\hline
\end{tabular}

of columns of data in the data file. Note that the first column of integers in the data file (Table 2) designates cases and is therefore not considered data. Line 3 of control information specifies for each variable whether or not it is to be rank transformed; for each variable in the data file, in the ordinal position corresponding to the data file column containing that variable, enter a 1 (yes) or 0 (no) to indicate whether or not that variable is to be rank transformed. This option is desirable for ordinal variables, since computing the nonparametric correlations on rank data ensures that no ordinal transformation will change the results. In the rank transformation, the standard procedure of assigning all ties the mean rank for those ties is used with the RankTransform routine in the program. In the example file, all five of the variables are rank transformed. Line 4 of control information specifies the matching tolerance assigned to each variable; the tolerance may be a real number. In the example, all five variables have a tolerance set at 3; if exact matches are desired, tolerance should be set to 0 . Line 5 of control information specifies the number of cases-that is, the number of rows of data in the data file. Line 6 of control information specifies the extent of output desired: the "summary" option outputs all three nonparametric correlations $\left(g, d_{y x}, e\right)$, their standard errors, and the ratio of each statistic to its standard error for comparison to the $z$ distribution in a significance test. The "full" option presents all the information of the summary output, plus information about the number of congruent, incongruent, and tied pairs in each analysis. Table 3 displays the summary output for the control file in Table 1 and data file in Table 2. Table 4 displays the full output corresponding to the same control and data files, except of course that the full output selection would have been specified. Notice that the output files use standard notation to designate partial correlations under the heading Correlation. The digit string 12.3, for example, indicates that variable 1 (column 1 of data in the data file) is the dependent variable, variable 2 (column 2) is the dependent variable, and variable 3 (column 3) is the only control variable. Similarly, the digit string 31.245 indicates that variable 3 is the dependent variable, variable 1 is the independent variable, with variables 2,4 , and 5 as control variables.

Line 7 of control information specifies the number of different correlations that are to be computed-that is, the number of distinct analyses. Table 1 specifies that six correlations are to be computed, and the six correlations desired are indicated in the following six lines. Notice that there are six corresponding lines of output in Table 3. Each line after line 7 specifies one correlation. The standard notation appearing in the control (and output) file to denote partial correlations must be used; that is, the first digit is the criterion variable, the second is the predictor, and digits following the period designate control variables. Each digit denotes a variable (column) in the data file. Because the $d_{y x}$ measure is asymmetric, the order of the first two integers is important: The first integer denotes $Y$ and the second denotes $X$. Line 8 in the example control file specifies the correlation between variables 1 and 2, where $Y$ is variable 1 and $X$ is variable 2 . Line 9 specifies the correlation between variables 1 and 2 with variable 3 partialed, while line 10 specifies the correlation between variables 1 and 2 with variables 3 and 4 both partialed, etc.

Each line in the summary output file (Table 3) contains all three nonparametric coefficients, their standard errors,

Table 3

Example Summary Output

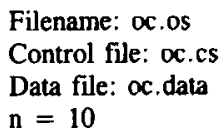

\begin{tabular}{lrcccccccc}
\multicolumn{3}{c}{$\begin{array}{c}\text { Goodman- } \\
\text { Kruskal }\end{array}$} & Sommers & Wilson & \multicolumn{3}{c}{ Standard Error } & \multicolumn{3}{c}{ Z Ratio } \\
Correlation & gamma & dyx & $\mathrm{e}$ & SEg & SEd & SEe & g/SEg & d/SEd & e/SEe \\
& & & & & & & & & \\
12 & -0.189 & -0.175 & -0.163 & 0.336 & 0.309 & 0.288 & -0.563 & -0.566 & -0.565 \\
12.3 & 0.176 & 0.167 & 0.158 & 0.486 & 0.462 & 0.443 & 0.363 & 0.361 & 0.357 \\
12.34 & 0.429 & 0.429 & 0.429 & 0.507 & 0.507 & 0.507 & 0.846 & 0.846 & 0.846 \\
12.345 & 1.000 & 1.000 & 1.000 & 0.000 & 0.000 & 0.000 & undefin & undefin & undefin \\
31.245 & 0.200 & 0.200 & 0.200 & 0.697 & 0.697 & 0.697 & 0.287 & 0.287 & 0.287 \\
54 & 0.056 & 0.047 & 0.044 & 0.327 & 0.275 & 0.263 & 0.170 & 0.169 & 0.169 \\
\hline
\end{tabular}


Table 4

Filename: $o c$.of

Control file: oc.cf

Data file: oc.dat

$\mathrm{n}=10$

Nonparametric Partial Correlations

\begin{tabular}{|c|c|c|c|c|c|c|c|c|c|}
\hline \multirow[b]{2}{*}{ Correlation } & \multirow{2}{*}{$\begin{array}{c}\text { Goodman } \\
\text { Kruskal } \\
\text { gamma }\end{array}$} & \multirow{2}{*}{$\begin{array}{c}\text { Sommers } \\
\text { dyx }\end{array}$} & \multirow{2}{*}{$\begin{array}{c}\text { Wilson } \\
\mathrm{e}\end{array}$} & \multicolumn{3}{|c|}{ Standard Error } & \multicolumn{3}{|c|}{ Z Ratio } \\
\hline & & & & SEg & SEd & SEe & $\mathrm{g} / \mathrm{SEg}$ & $\mathrm{d} / \mathrm{SEd}$ & $\mathrm{e} / \mathrm{SEe}$ \\
\hline 12 & -0.189 & -0.175 & -0.163 & 0.336 & 0.309 & 0.288 & -0.563 & -0.566 & -0.565 \\
\hline
\end{tabular}

Case numCon numInc numTieX numTieY numTieXY

$\begin{array}{rrrrrr}1 & 1 & 5 & 1 & 2 & 0 \\ 2 & 6 & 3 & 0 & 0 & 0 \\ 3 & 3 & 3 & 1 & 1 & 1 \\ 4 & 5 & 3 & 0 & 0 & 1 \\ 5 & 4 & 2 & 2 & 1 & 0 \\ 6 & 5 & 3 & 0 & 0 & 1 \\ 7 & 0 & 7 & 1 & 1 & 0 \\ 8 & 3 & 6 & 0 & 0 & 0 \\ 9 & 3 & 3 & 1 & 1 & 1 \\ 10 & 0 & 9 & 0 & 0 & 0 \\ & 30 & 44 & 6 & 6 & 4\end{array}$
12.3
0.176
0.167
0.158
0.486
0.462
0.443
$0.363 \quad 0.361 \quad 0.357$

Case numCon numlnc numTieX numTieY numTieXY

$\begin{array}{rrrrrr} & \text { num } & \text { numinc } & \text { num } & \text { num } & \text { num } \\ 1 & 1 & 3 & 1 & 0 & 0 \\ 2 & 5 & 2 & 0 & 0 & 0 \\ 3 & 3 & 0 & 0 & 0 & 1 \\ 4 & 3 & 0 & 0 & 0 & 1 \\ 5 & 2 & 1 & 0 & 1 & 0 \\ 6 & 3 & 0 & 0 & 0 & 1 \\ 7 & 0 & 2 & 1 & 1 & 0 \\ 8 & 0 & 2 & 0 & 0 & 0 \\ 9 & 3 & 0 & 0 & 0 & 1 \\ 10 & 0 & 4 & 0 & 0 & 0 \\ & 20 & 14 & 2 & 2 & 4\end{array}$

12.34

0.429

0.429

0.429

0.507

0.507

0.507

0.846

$0.846 \quad 0.846$

Case numCon numInc numTieX numTieY numTieXY

$\begin{array}{rrrrrr}1 & 1 & 1 & 0 & 0 & 0 \\ 2 & 2 & 0 & 0 & 0 & 0 \\ 3 & 1 & 0 & 0 & 0 & 1 \\ 4 & 2 & 0 & 0 & 0 & 0 \\ 5 & 2 & 1 & 0 & 0 & 0 \\ 6 & 1 & 0 & 0 & 0 & 0 \\ 7 & 0 & 0 & 0 & 0 & 0 \\ 8 & 0 & 0 & 0 & 0 & 0 \\ 9 & 1 & 0 & 0 & 0 & 1 \\ 10 & 0 & 2 & 0 & 0 & 0 \\ & 10 & 4 & 0 & 0 & 2\end{array}$

12.345

1.000

1.000

1.000

0.000

0.000

0.000

undefin undefin undefin

$\begin{array}{ccrrrr}\text { Case } & \text { numCon } & \text { numlnc } & \text { numTieX } & \text { numTieY } & \text { numTieXY } \\ 1 & 1 & 0 & 0 & 0 & 0 \\ 2 & 1 & 0 & 0 & 0 & 0 \\ 3 & 0 & 0 & 0 & 0 & 1 \\ 4 & 0 & 0 & 0 & 0 & 0 \\ 5 & 1 & 0 & 0 & 0 & 0 \\ 6 & 1 & 0 & 0 & 0 & 0 \\ 7 & 0 & 0 & 0 & 0 & 0 \\ 8 & 0 & 0 & 0 & 0 & 0 \\ 9 & 0 & 0 & 0 & 0 & 1 \\ 10 & 0 & 0 & 0 & 0 & 0 \\ & 4 & 0 & 0 & 0 & 2\end{array}$


Table 4 (Continued)

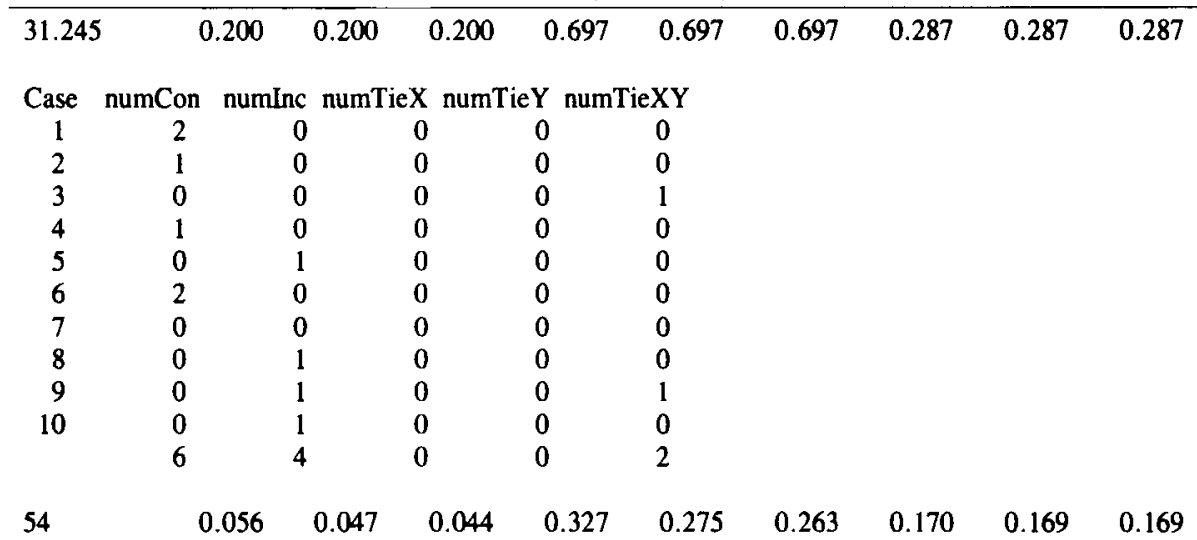

\begin{tabular}{crrrrr} 
Case & numCon & numinc & numTieX & numTieY & numTieXY \\
1 & 4 & 4 & 0 & 1 & 0 \\
2 & 5 & 0 & 1 & 3 & 0 \\
3 & 1 & 5 & 0 & 3 & 0 \\
4 & 4 & 4 & 1 & 0 & 0 \\
5 & 4 & 5 & 0 & 0 & 0 \\
6 & 5 & 4 & 0 & 0 & 0 \\
7 & 9 & 0 & 0 & 0 & 0 \\
8 & 2 & 5 & 1 & 1 & 0 \\
9 & 1 & 4 & 1 & 3 & 0 \\
10 & 3 & 3 & 0 & 3 & 0 \\
& 38 & 34 & 4 & 14 & 0 \\
\hline
\end{tabular}

and their $z$ ratios, for a given correlation. The full output file (Table 4) contains both the summary information and information about the number of concordant, discordant, and tied pairs of cases, per case. For example, Table 4 shows that all three coefficients are identical whenever there are no ties on $x$ only or $y$ only, and that the overall numbers of congruent and incongruent pairs decrease as the number of control variables increases. The latter result is clearly due to the increasing stringence with which pairs are screened for relevance, as defined above, as the number of control variables increases. The full output format might be useful in Monte Carlo studies or in checking the basis for curious results or discrepancies between the three nonparametric measures, and so forth.

A listing of the program and sample input and output files may be obtained from the author, Carl Craig Morris, Behavioral Statistics Program, Department of Psychology, Baylor University, Waco, TX 76798. To obtain a copy of the program, send a check for $\$ 3.50$ to the author. The check should be written to Baylor University. Listings of the program may also be obtained by contacting the author via electronic mail. The address is MorrisC@Baylor.BITNET.

\section{REFERENCES}

Asher, H. B. (1983). Causal modeling (2nd ed.). Beverly Hills, CA: Sage.

COHEN, J., \& CoHEN, P. (1983). Applied multiple regression/correlation analysis for the behavioral sciences (2nd ed.). Hillsdale, NJ: Erlbaum.

Davison, M. L., \& Sharma, A. R. (1988). Parametric statistics and levels of measurement. Psychological Bulletin, 104, 137-144.
GaITo, J. (1980). Measurement scales and statistics: Resurgence of an old misconception. Psychological Bulletin, 87, 564-567.

GoOdMan, L. A., \& KrUSKAL, W. H. (1954). Measures of association for cross classifications. Joumal of the American Statistical Association, 49, 732-764.

KIM, J. O., \& MUELLER, C. W. (1978). Introduction to factor analysis. Beverly Hills, CA: Sage.

Michell, J. (1986). Measurement scales and statistics: A clash of paradigms. Psychological Bulletin, 100, 398-407.

MoRsus, C. C. (1990a). Causal analysis shows retrieval may influence confidence in comprehension judgments. Manuscript submitted for publication.

MoRRIs, C. C. (1990b). Retrieval processes underlying confidence in comprehension judgments. Journal of Experimental Psychology: Leaming, Memory, \& Cognition, 16, 223-232.

NELSON, T. O. (1984). A comparison of current measures of the accuracy of feeling-of-knowing predictions. Psychological Bulletin, 95 , 109-133.

QuADE, D. (1974). Nonparametric partial correlation. In H. M. Blalock, Jr. (Ed.), Measurement in the social sciences (pp. 369-396). Chicago: Aldine.

REYNOLDS, H. T. (1974). Ordinal partial correlation and causal inferences. In H. M. Blalock, Jr. (Ed.), Measurement in the social sciences (pp. 399-423). Chicago: Aldine.

Somers, R. H. (1962). A new asymmetric measure of association. American Sociological Review, 27, 291-292.

TOWNSEND, J. T., \& AshBY, F. G. (1984). Measurement scales and statistics: The misconception misconceived. Psychological Bulletin, 96, 394-401.

WILson, T. P. (1974). Measures of association for bivariate ordinal hypotheses. In H. M. Blalock, Jr. (Ed.), Measurement in the social sciences (pp. 343-368). Chicago: Aldine.

(Manuscript received September 18, 1989; revision accepted for publication January 4, 1990.) 